Acta Crystallographica Section A

Foundations of Crystallography

ISSN 0108-7673

\section{A new vision for Acta Crystallographica Section A}

\author{
Simon J. L. Billinge ${ }^{\mathrm{a} *}$ and Jianwei Miao ${ }^{\mathrm{b} *}$
}

a Department of Applied Physics and Applied Mathematics, Columbia University, 200 Mudd, 500 W 120th Street, New York, NY 10027, USA, and ${ }^{\mathbf{b}}$ Department of Physics and Astronomy and California NanoSystems Institute, University of California, Box 951547, Los Angeles, CA 900951547, USA

The impact of crystallography is profound. A detailed knowledge of material structure is a prerequisite to any understanding of material properties, and materials are at the heart of just about every next-generation technology. For example, advanced complex materials hold the key to many solutions to our biggest problems in sustainable energy, health and the environment. Acta Crystallographica Section A (Acta A) is the foundation on which the whole suite of IUCr crystallography journals are built, containing as it does papers describing fundamental developments in crystallographic theory and practice. It reports developments in the method rather than the results of applying those methods and, as such, holds a special place in the firmament of the IUCr journals. The papers in Acta A are reasonably well cited (impact factor 2.24) but the impact of the work they describe has much farther reach, forming the basis of transformative discoveries reported in journals from Nature and Science all the way to specialized journals from chemistry and physics to planetary science and pharmaceuticals, with the other IUCr journals in between.

One hundred years on from the first experiments in X-ray crystallography, and as we approach the International Year of Crystallography in 2014, it is timely to revisit the state of crystallography and advances taking place in studies of material structure. The landscape in this regard looks very different from the way it did even ten years ago, and unrecognizable from 30 years ago, when synchrotron X-ray sources first came onto the scene, from the methods being used to probe the structure, to the computing resources that are now available, to the very nature of the materials-structure questions being studied. One of the greatest challenges is the study of nanostructures: far from the crystallographic ideal of an infinite periodically repeating lattice, but of fundamental importance in applications such as battery electrodes, catalytic stacks and cosmetics. Is this crystallography? One would have to say yes. Material properties depend sensitively on defects, but many functional materials, fast-ion conductors being a case in point, have so many defects that it is hard to distinguish what is defect and what is structure; the defects are intrinsic and not a materials-processing-dependent microstructural issue more at home in Acta Materiala. On the sources side, X-ray free-electron lasers (X-FELs) are currently under rapid development worldwide; they produce intense X-ray pulses with a peak brilliance a billion times higher than synchrotron radiation and a pulse duration as short as a few femtoseconds. These X-FELs are expected to probe the structure and dynamics of materials at the space and time limit. On the methods side, three-dimensional atomic resolution imaging is just over the horizon, even for non-periodic materials such as defects and dislocations in nanostructures. As one can see, researchers are responding to these challenges and advances with an explosion of creativity. Traditional crystallography, as applied to periodic crystals, continues to be a rich and dynamic field. However, for Acta $A$ to overlook the exciting developments in crystallography more broadly defined above would be folly and would risk it 
losing its pre-eminent position as the premier journal describing foundations in structure science.

To address this changing landscape, in consultation with the the Editorial Board of the IUCr journals, we are making some changes to Acta A. First, to reflect the exciting nature of materials-structure research, we will change the name of Section A from Foundations of Crystallography to Foundations and Advances. Today's advances become tomorrow's foundations, and the new name reflects our excitement at emerging developments in the field and our interest in reporting them. More importantly, we will create a new Advances section within Section A. Manuscripts in the Advances section will be 'fast-tracked' for rapid publication. They will receive the same rigorous review and editorial oversight, but will be dealt with in a special process with a target time from submission to publication of 6-8 weeks. Certain papers in the Advances section will be highlighted. For these, viewpoint articles will be commissioned, describing the novelty and impact of the advance, and short news releases for a general audience will be written and released in conjunction with the publication of the paper. These things will make Acta $A$ an attractive place to publish high-impact work that focuses on structural science, with it appearing quickly and being widely disseminated, but built on the tradition and reputation that comes with Acta A. Articles describing original research of high potential impact may be submitted for consideration for the Advances section of Acta A. Papers submitted as regular articles may also be selected by the editors for fast tracking in the Advances section. Finally, Advances review articles will be commissioned on an occasional basis, periodically capturing the state of play in an emerging area of structure science. We are accepting papers for the Advances section now, though the first such article will not appear until the beginning of 2014. We strongly encourage you to send your most exciting work for consideration. Looking forward, crystallography in the next century (beyond Bragg) will be as least as exciting as during the past one. Acta $A$ will continue to be a leading journal reporting fundamental advances in all areas of crystallography. 